\title{
CO-INCIDENCE OF MICROFILARIA \& TOXOPLASMA IN LYMPH NODE -A RARE DIAGNOSIS BY FINE NEEDLE ASPIRATION CYTOLOGY (FNAC)
}

\author{
Dilip Chandra Barman, Sanjay Kumar Mallick, Bidyut Krishna Goswami, Sweta Sarawagi,
}

\author{
1. Assistant Professor, Department of Pathology, North Bengal Medical College, Darjeeling, West Bengal. \\ 2. Assistant Professor, Department of Microbiology, North Bengal Medical College, Darjeeling, West Bengal. \\ 3. Professor, Department of Pathology, N. R. S. Medical College, Kolkata, West Bengal. \\ 4. Pathologist, B. S. Diagnostic Centre, Siliguri, West Bengal, India.
}

\section{CORRESPONDING AUTHOR}

Dr. Dilip Chandra Barman

Department of Pathology,

North Bengal Medical College and Hospital,

Darjeeling, India.

E-mail: dilip77d@gmail.com,

$\mathrm{Ph}: 00919474327035$.

ABSTRACT: Toxoplasma lymphadenitis is a rare disease entity, microfilaria also rarely found in lymph node aspirate but coincidence of toxoplasma gondii and microfilaria in lymph node is very rare. We report here one such case of a four year old child presented with cervical lymphadenopathy, fine needle aspiration cytology showed microfilaria and bradyzoides of toxoplasma gondii, granuloma in a background of reactive lymphoid cells. On serology, serum IgM anti toxoplasma antibody was positive and IgG was negative.

KEY WORDS: Fine needle aspiration cytology (FNAC), Lymph node, Toxoplasma, Microfilaria.

INTRODUCTION: Filariasis is a major public health problem and it is endemic all over India. ${ }^{[1,2]}$ About 120 million people infected in this region and in need of treatment including 40 million people with overt disease. [3] Despite its high incidence, it is infrequent to find microfilaria in FNAC smears and only incidentally detected in various sites such as lymph node, breast lump, bone marrow, bronchial aspirate, pleural and pericardial fluid, ovarian cyst fluid in clinically unsuspected cases of filariasis with absence of microfilaria in the peripheral blood. [4] Microfilaria has been observed as a coincidental finding with other infective, inflammatory conditions and neoplastic lesions. We present here a very rare case in which microfilaria and Toxoplasma gondi (bradyzoides) together were observed in the fine needle aspirates of cervical lymph node. No such case report has been documented till date.

CASE REPORT:A 4-year-old male child belongs to a Nepali tribal community of low socioeconomic condition in Dooars region, presented with multiple enlarged right cervical lymph nodes since two months with flu like symptoms. On examination, the lymph nodes were at the right posterior triangle of neck, mildly tender, firm; together measuring $3.5 \mathrm{~cm} \times 3.2 \mathrm{~cm}$. Axillary and inguinal lymph nodes were not palpable. He had no visual or neurological symptom. FNAC of the lymph nodes were done. Aspirate material were whitish granular and microscopic examination of the air dried Leishman stained and alcohol fixed Hematoxylin \& Eosin (H\&E) stained smears showed small cluster of epithelioid histiocytes without necrosis associated with sheathed microfilaria of Wuchereria bancrofti and cyst of Toxoplasma gondii (bradyzoides) in a background of heterogeneous population of reactive lymphoid cells [Figure 1 \& 2]. Aggregates of activated large ovoid lymphoid cells also seen in the background [Figure 3]. 
Peripheral blood smear were prepared from blood sample taken for three consecutive nights showed no microfilaria but eosinophilia was noted (12\%). In urine examination, there was no evidence of chyluria and microfilaria in the centrifuged deposit. Serological study by Enzyme Linked Immunosorbent Assay (ELISA) for serum IgM anti toxoplasma antibody was positive but IgG was negative. Patient was non-reactive for HIV-1 \& 2 . All the test results were negative in patient's mother.

DISCUSSION: Microfilaria is transmitted by the Culex mosquito and is caused by two closely related nematodes, Wuchereria bancrofti and Brugia malayi. About 95\% of cases lymphatic filariasis is caused by infection with Wuchereria bancrofti. [1] Infective larvae penetrate through the skin wound, enter the lymphatics and travel to the lymph nodes. Adult worms are thread like creamy white. Females are viviparous, once fertilized they discharge as many as 50,000 microfilaria per day. [5] The sheathed microfilaria of Wuchereria bancrofti having multiple, coarse, discrete nuclei extending from the head to the tail, except in the small terminal portion of the caudal end. Thus species are identified by seeing the larval form. ${ }^{[2]}$ Microfilaria displays a nocturnal periodicity; this is basically a biological adaptation to the nocturnal biting habit of vector mosquitoes. That is why at least three consecutive night blood sample were examined. The clinical manifestations varies, may be asymptomatic, or may have acute or chronic manifestations e.g. lymphangitis, lymphadenitis, lymphedema, and elephantiasis. [1]

Toxoplasmosis is cosmopolitan zoonotic infection caused by a single-celled obligate intracellular parasite called Toxoplasma gondii. It exists in three form e.g. oocysts, tachyzoites and bradyzoides (tissue cysts) and transmitted by eating vegetables or drinking water contaminated with the oocysts commonly passed by cats or from ingestion of mutton, pork, chicken etc. Early maternal infection (first and second trimester) results in spontaneous abortion and intrauterine foetal death (IUFD).

Toxoplasma is an infection which is highly variable in its clinical manifestation, these include myocarditis, encephalomyelitis, chorioretinitis especially in the immunocompromised host and the healthy host who harbour the infection usually remains asymptomatic.

Superficial enlarged lymph nodes are the most common presenting sign of acquired toxoplasmosis. [6] Usually involves the posterior cervical lymph nodes. [7] In cytology smear, some lymphoid cells with relatively large, ovoid, pale nuclei may be seen. These cells probably correspond to the pale monocytoid B-cells in histological sections. Microcysts and organisms of Toxoplasma gondii are rarely seen. Diagnosis is usually done by identifying tissue cysts and serological tests. [6], [8],[9], [10]

Here in our case also, right posterior cervical lymph nodes were involved and the smears showed loose aggregates of activated large ovoid lymphoid cells [Figure 3].

The objective of the study is to document the value FNAC in the diagnosis of filariasis and toxoplasmosis in lymph node and thus demonstration and identification of these parasites in cytological smears played a significant role in the prompt recognition of the disease and initiation of specific treatment, thus avoiding the more severe manifestations of lymphatic filariasis and toxoplasmosis. Secondly to highlight such a rare coincidence of two infection in lymph node.

CONCLUSION: Although microfilaria and toxoplasma cysts in cytological smears are considered incidental findings, this case illustrates the value of FNAC in the detection of asymptomatic and clinically unsuspected cases of filariasis \& toxoplasmosis. Absence of microfilaria in the 
peripheral blood does not exclude filarial infection. Filariasis may be detected in a clinically unsuspected case, especially in an endemic zone. The spectrum of host response may vary from no reaction to a marked inflammatory response. ${ }^{[1]}$ The entire spectrum of changes should be kept in mind while practicing cytopathology in an endemic area. In such situations, a strong clinical suspicion and meticulous screening of cytology smears may lead to a correct diagnosis. At the same time, the cytopathologist should search for a coexisting pathology thoroughly. In the present case, we feel that the presence of microfilaria in association with Toxoplasma gondii is a very rare coincidental finding. The patient was harboring subclinical filariasis when he was suffering from toxoplasmosis. Microfilaria was not detected in the peripheral blood smears. This also highlights the importance of screening smears for parasites even in the absence of clinical symptoms, particularly in highly endemic areas.

\section{REFERENCES:}

1. Park K. Park's Textbook of Preventive and Social Medicine. 21 ${ }^{\text {st }}$ ed. Jabalpur, India: Banarasidas Bhanot publisher; 2011.Chapter 5; page: 244.

2. Chatterjee KD. Parasitology (Protozoology and Helminthology) $12^{\text {th }}$ ed. Calcutta, India: Chatterjee Medical Publishers; 1980; 190-8.

3. WHO (2009), Weekly Epidemiological Record, No. 11/12, 13th March, 2009.

4. Varghese R, Raghuveer CV, Pai MR, Bansal R. Microfilariae in cytologic smears: A report of six cases. Acta Cytol 1996; 40 (2): 299-30.

5. Nelson GS. Filarial infections. Med Int 1981; 1: 77-82.

6. Stansfelt. AG. Histological diagnosis of Toxoplasmic lymphadenitis. J. clin. Path 1961; 14: 565.

7. Leopold GK. Koss' Diagnostic Cytology and its Histopathologic Bases: $5^{\text {th }}$ ed, Lippincott Williams \& Wilkins Publisher; 2006. Vol-II, Chapter 31, Page: 1195.

8. Argyle JC, Schumann GB, Kjeldsberg CR, Anthens JW. Identification of a Toxoplasma cyst by fine needle aspiration. Am J Clin Pathol 1983; 80: 256-258.

9. Christ ML, Feltes-Kennedy M. Fine needle aspiration cytology of Toxoplasmic lymphadenitis. Acta Cytol 1982; 26: 425-428.

10. Jayaram N, Ramprasad AV, Chatan M, Sujay AR. Toxoplasma lymphadenitis. Analysis of cytologic and histologic criteria and correlation with serologic tests. Acta Cytol 1997; 41: 653-658.

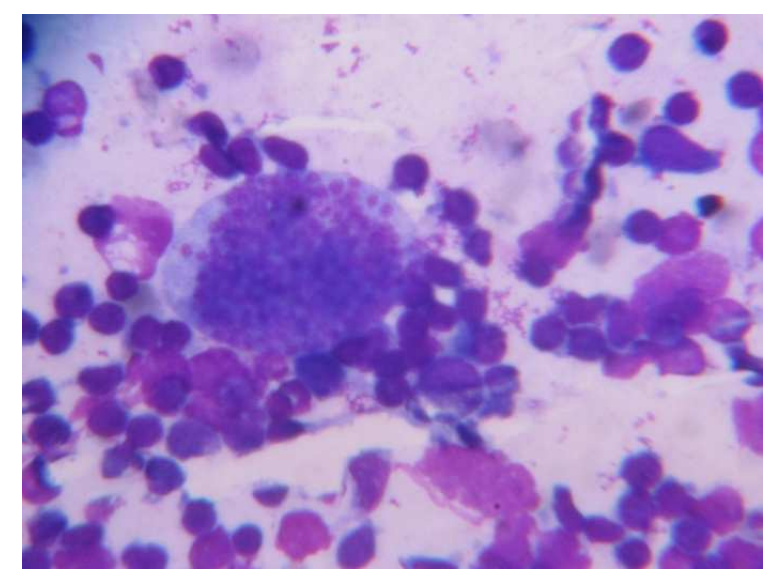

Figure 1: Tissue cyst of Toxoplasma gondii (Leishman's stain $x$ 40) 


\section{CASE REPORT}

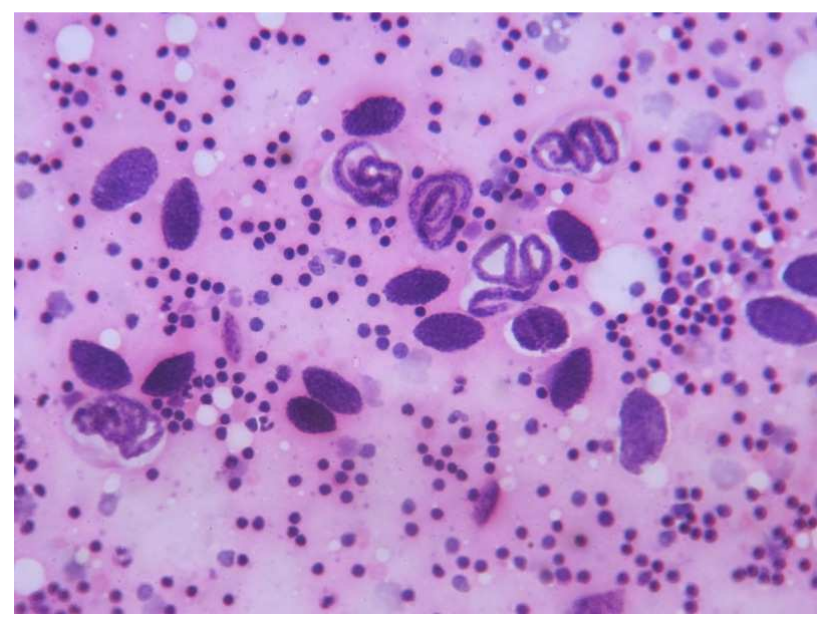

Figure 2: Tissue cysts Toxoplasma gondii \& coiled microfilaria of Wuchereria bancrofti (H/E stain $\mathrm{x10})$

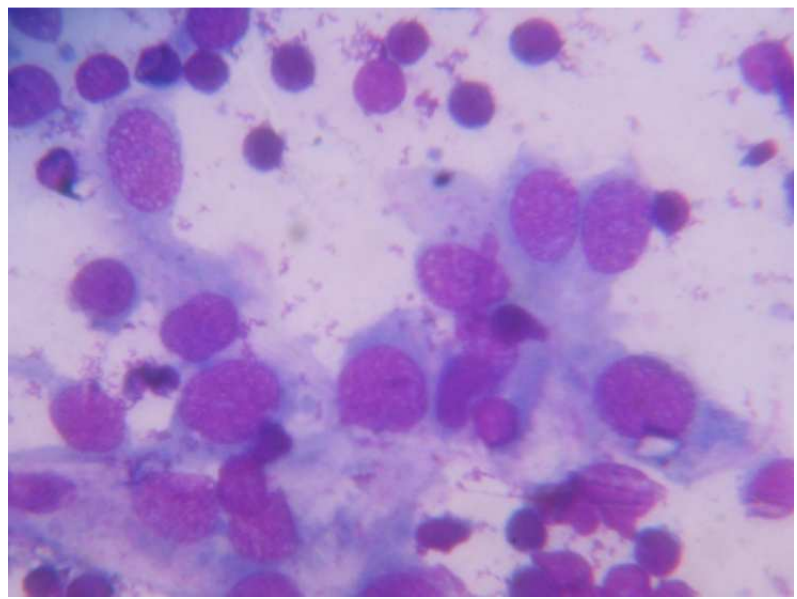

Figure 3: Activated large ovoid lymphoid cells (Leishman's stain $x$ 100) 